\title{
Editorial
}

\section{Apoptosis in viral pathogenesis}

\author{
JM Hardwick ${ }^{\star 1,2,3,4,5}$ \\ 1 Department of Molecular Microbiology and Immunology, Johns Hopkins \\ University Schools of Public Health and Medicine, Baltimore, Maryland 21205, \\ USA \\ 2 Department of Neurology, Johns Hopkins University Schools of Public Health \\ and Medicine, Baltimore, Maryland 21205, USA \\ ${ }^{3}$ Department of Pharmacology and Molecular Sciences, Johns Hopkins \\ University Schools of Public Health and Medicine, Baltimore, Maryland 21205, \\ USA \\ ${ }^{4}$ Department of Biochemistry and Molecular Biology, Johns Hopkins University \\ Schools of Public Health and Medicine, Baltimore, Maryland 21205, USA \\ ${ }^{5}$ Department of Oncology, Johns Hopkins University Schools of Public Health \\ and Medicine, Baltimore, Maryland 21205, USA \\ * Corresponding author: JM Hardwick, Department of Molecular Microbiology \\ and Immunology, Johns Hopkins University Schools of Public Health and \\ Medicine, Baltimore, MD 21205, USA. E-mail: hardwick@jhu.edu
}

It is difficult if not impossible for a virus to infect a cell and not trigger the cell's 'burglar' alarm, activating the cellular programmed cell death pathway. Early discoveries with viruses taught us a great deal about both viral and cellular death pathways. Eileen White and colleagues identified viral apoptosis-inhibitory genes by studying adenovirus mutants with an unusual deg (degradation) phenotype, ${ }^{1,2}$ leading to an understanding of how E1b $55 \mathrm{~K}$ and E1b $19 \mathrm{~K}$ inhibit apoptotic cell death by distinct mechanisms. Lois Miller's laboratory made a link to virus-induced disease when they demonstrated that the baculovirus-encoded caspase inhibitor P35 was required for pathogenesis..$^{3,4}$ Jeurissen et al. first associated virus-induced apoptosis with disease in chickens when they showed that chicken anemia virus infection of hatchlings resulted in thymocyte apoptosis. ${ }^{5}$ Cellular anti-apoptotic genes were then found to convert a lytic virus infection to a persistent infection, perhaps explaining the long-term persistence of alphaviruses in the brain. ${ }^{6}$ Early in the apoptosis field, a number of laboratories gathered evidence for HIV-triggered apoptosis in AIDS. ${ }^{7}$ Several years and many genes later, it is surprising how little we understand about the role of apoptotic cell death in viral disease mechanisms. For example, the role of viral $\mathrm{Bcl}-2$ and other virus-encoded apoptosis regulatory proteins encoded by herpes viruses have been studied in some detail but the mechanisms by which these genes might alter herpesvirus-associated disease and tumorigenesis is unknown. It turns out that the issues are complex and these are difficult questions to answer.

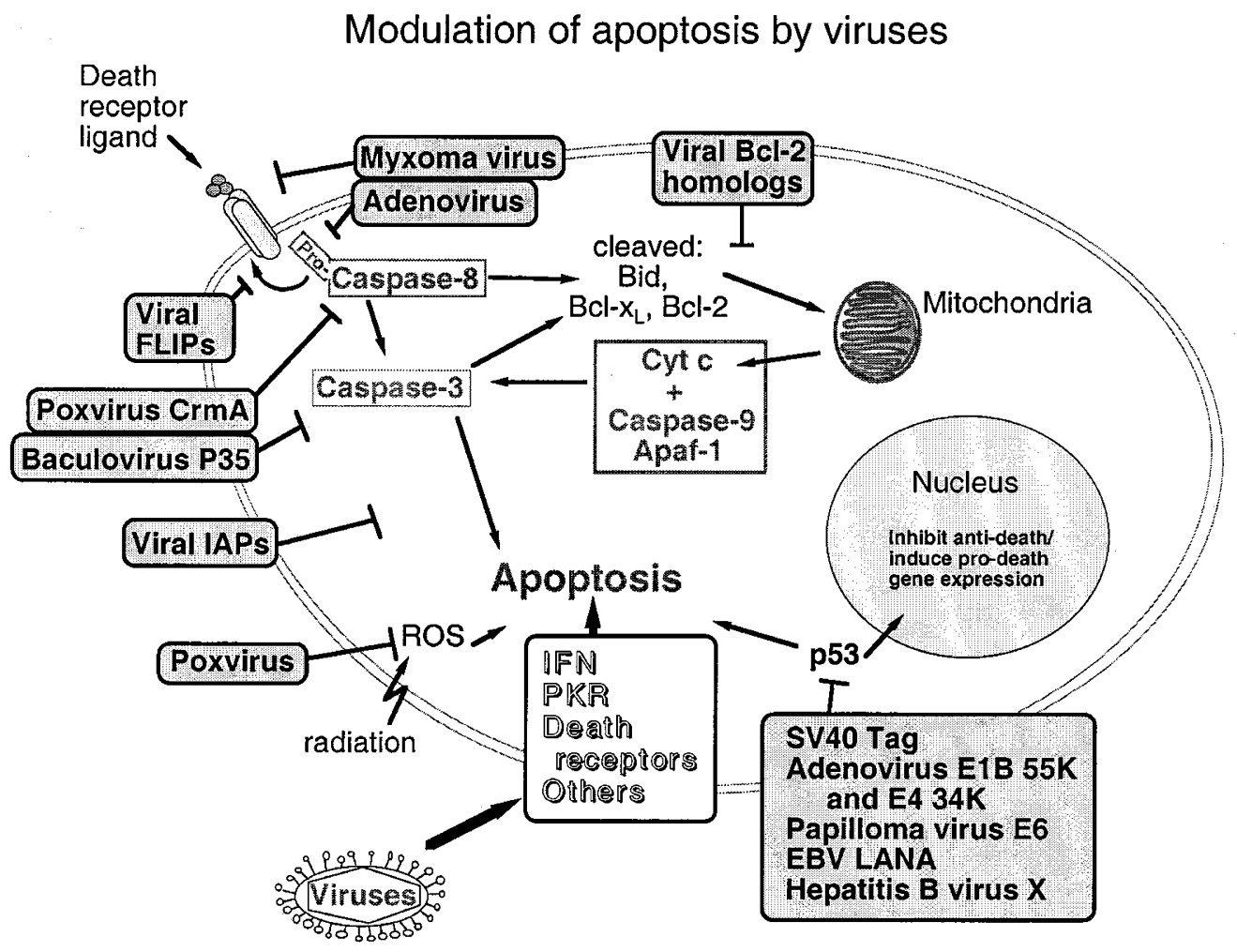

Figure 1 Modulation of apoptosis by viruses 
Adenovirus takes the prize for having the greatest number of identified gene products that induce or inhibit programmed cell death. ${ }^{8-10}$ Many other viruses have now been shown to trigger apoptotic cell death and/or encode inhibitors of apoptosis. ${ }^{11,12}$ Viruses interfere with programmed cell death from the beginning of the cell death pathway to the end (shaded boxes in Figure 1). One of the mechanisms by which myxoma virus (a poxvirus) modulates the cellular response to infection is encoding viroceptors or virokines that mimic cellular receptors and cytokines. ${ }^{13} \mathrm{M}-\mathrm{T} 2$ of myxoma virus is a secreted tumor necrosis factor receptor homologue. ${ }^{14}$ At the distal end of the pathway, the baculovirus AcMNPV and cowpox virus encode protease inhibitors that prevent cellular caspases from terminating the cell. ${ }^{15,16}$ Although many of these viral factors have been studied in great molecular detail, it is less clear how these factors alter a disease state.

The following three review articles deal with various aspects of apoptosis in virus infections with special emphasis on disease pathogenesis. Glen Barber considers cellular defense mechanisms against viruses including cytotoxic lymphocytes and cytokines. Interferon, interferon-induced genes and interferon regulatory factors (IRFs) modulate apoptosis of virus-infected cells. But viruses also manipulate these host cell defense mechanisms. The use of knockout and transgenic animals has helped determine the roles of these host factors in viral infections.

Terry Finkel (HIV) and Rollie Clem (baculoviruses) discuss individual viruses and their effects on host cells and host organisms. Baculoviruses trigger apoptosis of insect cells by several possible mechanisms, and inhibit apoptosis by encoding either an IAP protein or P35. These inhibitors are required to kill caterpillars, but there is an exception when the host ( $T$. ni) provides a compensating function. AIDS patients die from the consequences of lymphocyte cell death. But the mechanisms by which HIV triggers the death of uninfected cells in AIDS patients is still a mystery that is beginning to unfold.
We wish to dedicate this set of reviews to the memory of Lois Miller as a small tribute to her pioneering work in this area.

\section{References}

1. White $E$, Grodzicker T and Stillman BW (1984) Mutations in the gene encoding the adenovirus early region 1B 19,000-molecular weight tumor antigen cause the degradation of chromosomal DNA. J. Virol. 52: 410-419

2. White E, Cipriani R, Sabbatini P and Denton A (1991) Adenovirus E1B 19kilodalton protein overcomes the cytotoxicity of E1A proteins. J. Virol. 65: 29682978

3. Clem RJ, Fechheimer M and Miller LK (1991) Prevention of apoptosis by a baculovirus gene during infection of insect cells. Science 254: 1388-1390

4. Clem RJ and Miller LK (1993) Apoptosis reduces both the in vitro replication and the in vivo infectivity of a baculovirus. J. Virol. 67: 3730-3738

5. Jeurissen SHM, Wagenaar F, Pol MA, van der EB AJ and Noteborn MHM (1992) Chicken anemia virus causes apoptosis of thymocytes after in vivo infection and of cell lines after in vitro infection. J. Virol. 66: 7383-7388

6. Levine B, Huang Q, Isaacs JT, Reed JC, Griffin DE and Hardwick JM (1993) Conversion of lytic to persistent alphavirus infection by the bcl-2 cellular oncogene. Nature (London) 361: 739-742

7. Ameisen JC, Estaquier J, Idziorek T and De Bels F (1995) The relevance of apoptosis to AIDS pathogenesis. Trends Cell Biol. 5: 27-32

8. White $E$ (1998) Regulation of apoptosis by adenovirus $E 1 A$ and $E 1 B$ oncogenes. Semin. Virol. 8: 505-513

9. White $E$ (1996) Life, death, and the pursuit of apoptosis. Genes Dev. 10: 1-15

10. Roulston A, Marcellus RC and Branton PE (1999) Viruses and apoptosis. Annu. Rev. Microbiol. 53: 577-628

11. Hardwick JM (1997) Virus-induced apoptosis. Adv. Pharmacol. 41: 295-336

12. Hardwick JM (1998) Viral interference with apoptosis. Semin. Cell Dev. Biol. 9: 339-349

13. NashP, BarrettJ, Cao JX, Hota-Mitchell S, Lalani AS, EverettH, XuXM, RobichaudJ, HnatiukS, AinslieC, SeetBT and McFadden G (1999) Immunomodulation by viruses: the myxoma virus story. Immunol. Rev. 168: 103-120

14. Xu X, Nash P and McFadden G (2000) Myxoma virus expresses a TNF receptor homolog with two distinct functions. Virus Genes 21: 97-109

15. Clem RJ, Hardwick JM and Miller LK (1996) Anti-apoptotic genes of baculoviruses. Cell Death Differ. 3: 9-16

16. Ray CA, Black RA, Kronheim SR, Greenstreet TA, Sleath PR, Salvesen GS and Pickup DJ (1992) Viral inhibition of inflammation: cowpox virus encodes an inhibitor of the interleukin-1-beta converting enzyme. Cell 69: 597-604 